\title{
Variant Forms of Primary Aldosteronism: Reconsideration of the Differential Diagnosis and Treatment
}

Primary aldosteronism is the syndrome resulting from the secretion of excessive amounts of aldosterone. It is clinically characterized by a combination of hypertension, hypokalemia, increased plasma aldosterone, and suppressed plasma renin (1). The wide availability of the sensitive aldosterone assay and of adrenal imaging techniques have made it much easier to make the diagnosis. Although the incidence has been reported to be less than $0.5 \%$ of all hypertensive patients, it is now one of the most representative causes of surgically curable endocrine hypertension. Therefore, there is no question about the importance of making correct diagnosis.

The underlying adrenal disease is usually a unilateral solitary adenoma (APA) and could be cured by unilateral adrenalectomy. However, bilateral adrenal hyperplasia referred as idiopathic hyperaldosteronism (IHA) is seen in about onequarter of all cases. In addition, evidence has accumulated to indicate the presence of variant forms: aldosterone-producing renin-responsive adenoma (AP-RA), primary adrenal hyperplasia (PAH), unilateral hyperplasia, and multiple APA. The type of adrenal pathology must be ascertained very carefully to determine the most appropriate type of therapy: surgical removal for APA and medical treatment for IHA.

See also p 970.

Both computed tomography $(\mathrm{CT})$ and magnetic resonance imaging (MRI) are now equally sensitive and accurate when the tumor size is larger than $10 \mathrm{~mm}$ in diameter (2). These imaging methods however may not detect an adenoma smaller than 5 $\mathrm{mm}$ in diameter. If bilateral enlargement of the adrenals is detected, it is generally diagnosed as IHA and is not operated. However, it should be noted that the criteria of the adrenal hyperplasia in imaging techniques have not been fully established due to the variance in the size of the normal adrenals. In addition, IHA is not necessarily accompanied by the anatomical enlargement of the adrenals. IHA may be mistakenly diagnosed as APA due to the nodular lesion often seen in the hyperplastic portion. On the contrary, APA may be mistakenly diagnosed as IHA because of the coexisting hyperplastic change and/or bilateral nodularity of the non-adenoma portion. The diagnosis of IHA therefore can be confirmed only after histological examination of the removed adrenals. Morphological findings by CT/MRI should be confirmed by further studies which provide data of function and localization to ensure the pathology of the lesion and the diagnosis.

One of the most commonly performed maneuvers is the upright posture test (3). The premise is that APA is unresponsive to a slight change in angiotensin II (renin) but is sensitive to ACTH showing diurnal rhythm, while IHA is angiotensin IIresponsive. The discrimination value of the test is $85 \%$ if the test is validated by the lack of increase in plasma cortisol (4). However, there are two exceptions: AP-RA which is angiotensin II-responsive (5) and PAH which is angiotensin IIunresponsive (5). AP-RA can be cured by adrenal surgery. Although it is reported that the aldosteronism is relieved by removal of one hyperplastic gland in $\mathrm{PAH}$, it is difficult to determine which side of the adrenals should be removed.

Plasma 18-OH-B as precursor of aldosterone and urinary excretion of 18-oxo-cortisol and 18-OH-cortisol as hybrid steroids have been demonstrated to be increased in APA and are useful in differentiating APA from IHA. However, these steroids can be measured only in limited laboratories.

Advances in CT and MRI relegated adrenal scintigraphy to infrequent use. Yet the method is meritorious in ascertaining the function and localization as well, especially if combined with dexamethasone suppression. The over all discrimination value between APA and IHA is about 90\% (6). Adrenal scintigraphy should be indicated when laterality of the lesion is not identified by CT/MRI, although small and/or functionally weak adenomas may not be visualized.

Adrenal venous sampling is an invasive but a powerful procedure to ascertain the function as well as the laterality of the lesion. The reported discrimination value is as high as $95 \%$ (6) if the blood sampling from both sides of the adrenal vein is accomplished. It should be reminded, however, that the failure rate of collecting blood samples from both sides is as high as $26 \%$ (6). The procedure is indicated when the laterality of the lesion is not determined by CT/MRI.

Algorithm of determining the subsets and choice of therapy in patients with confirmed primary aldosteronism is shown in Fig. 1. The principle is to match the morphology and function for the correct diagnosis. We start from the CT/MRI as the first line localization technique to determine the morphological type. The next step is the posture test to determine the functional type. If the lesion is morphologically unilateral and functionally angiotensin II-unresponsive, the diagnosis of APA can be made. However, if the functional type is angiotensin II-responsive, adrenal scintigraphy/adrenal venous sampling as second line localization technique should be performed to distinguish AP-RA from IHA with nodule(s). The identification of a unilaterally hyperfunctioning adrenal is a diagnostic point.

If morphologically not unilateral (bilateral or equivocal) by 


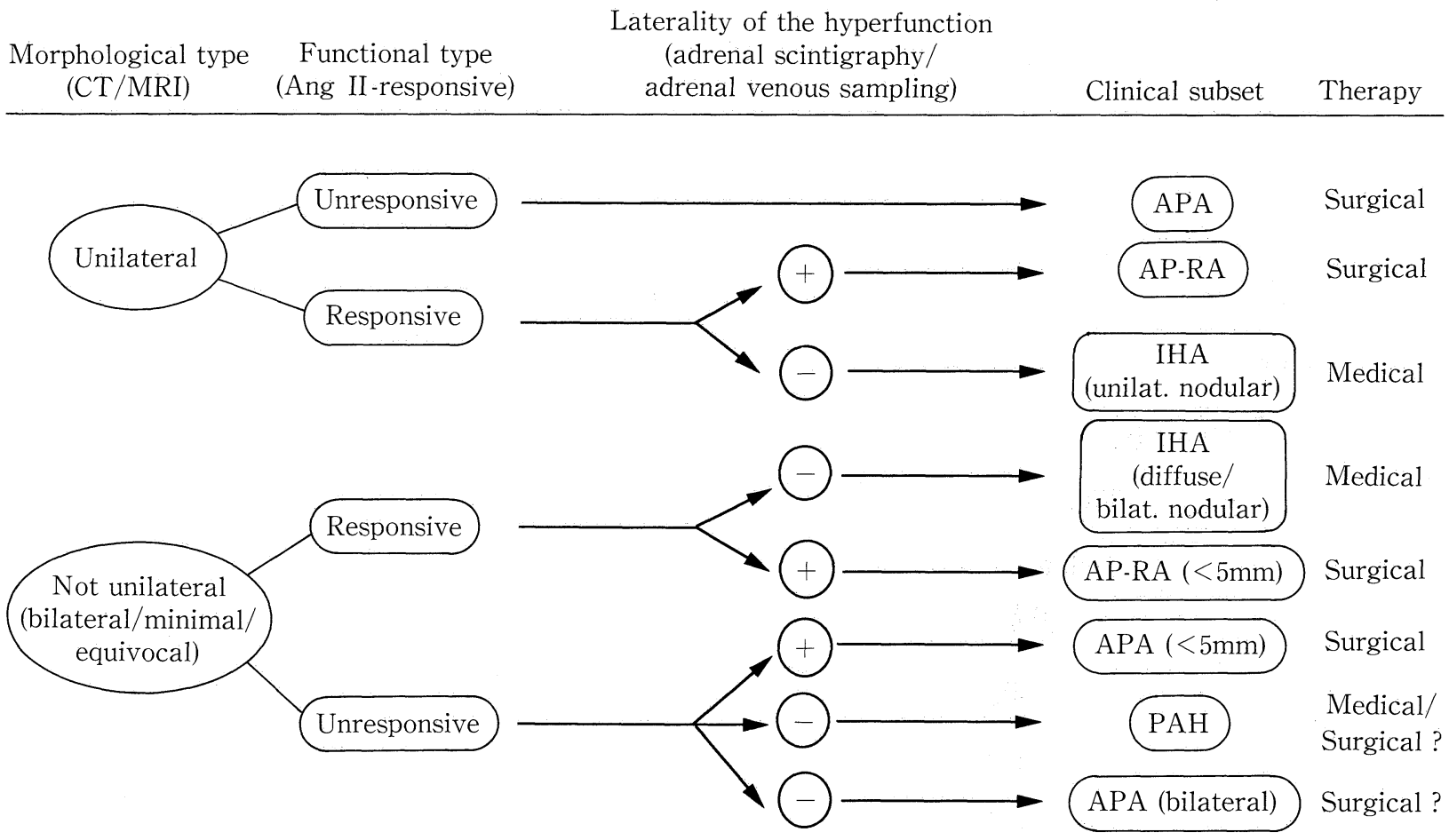

Figure 1. A step-wise diagram for the differential diagnosis of variant forms of primary aldosteronism and recommended type of therapy.

CT/MRI, the second line localization technique should be performed in addition to the posture test. Although most of the angiotensin II-responsive cases are IHA, small AP-RA is not completely excluded. If laterality of the hyperfunction is demonstrated by the second line localization technique in cases which are morphologically not unilateral and functionally angiotensin II-unresponsive, small APA is most likely. By contrast, if both sides are equally hyperfunctioning, the definition of PAH may be used. It is recommended to repeat the hormone assay and first line localization technique every 6 to 12 months when hyperfunction of any side of the adrenal is not identified.

As evident from Fig. 1, the type of therapy is determined based on the laterality of the lesion: surgery for unilateral lesion and medical therapy for bilateral lesion. However, Matsuda et al (7) reported a case of primary aldosteronism with bilateral multiple adenomas. The report is quite intriguing academically and clinically. First of all, the diagnosis of multiple adenomas was immunohistochemically proved. Enzymes related to aldosterone synthesis were found only in the tumor portion but not in the adjacent hyperplastic zona glomerulosa, excluding the diagnosis of IHA. It is suggested that multiple APA is essentially different from IHA with nodularity. Secondarily, bilateral partial adrenalectomy was successfully performed to correct the electrolyte balance and hypertension. The results therefore provide evidence for a possible cure of hyperaldosteronism by surgical therapy even if the lesion is bilateral. However, although preoperative endocrine studies showed the adenoma pattern, the diagnosis of multiple APA was made histologically after surgery. We can not extend the indication of the adrenal surgery to the bilateral lesion unless hyperfunction of the non-tumor portion is preoperatively excluded.

The diagnosis and treatment of primary aldosteronism are generally well established. However, we should remember the fact that there are variant forms with morphological and/or functional aberrations. In order to ensure the choice of treatment, further studies to integrate the anatomical and functional data in a larger number of patients are required.

Mitsuhide NAruse, MD $^{1)}$, Hiroshi Demura, MD ${ }^{2)}$, Kiyoko NaruSE, $\mathrm{MD}^{3)}$, Akiyo TANABE, $\mathrm{MD}^{3)}$, ToshiRou Seki, $\mathrm{MD}^{3)}$ and Takanobu Yosнiмoto, $\mathrm{MD}^{4)}$ Associate Professor ${ }^{1}$, , Professor ${ }^{2}$, Clinical Instructor ${ }^{3}$ of Medicine and Research Fellow of the Japan Society for Promotion of Science ${ }^{4}$, The Second Department of Medicine, Tokyo Women's Medical College, 8-1 Kawadacho, Shinjuku-ku, Tokyo 162

\section{References}

1) Conn JW. Primary aldosteronism, a new clinical syndrome. J Lab Clin Med 43: 3, 1955.

2) Gross MD, Shapiro B. Scintigraphic studies in adrenal hypertension. Semin Nucl Med 19: 122, 1989.

3) Ganguly A, Dowdy AJ, Luetscher JA, Melada GA. Anomalous postural response of plasma aldosterone concentration in patients with aldosterone-producing adrenal adenoma. J Clin Endocrinol Metab 36: 401, 1973.

4) Fontes RG, Kater CE, Biglieri EG, Irony I. Reassessment of the predictive value of the postural stimulation test in primary aldosteronism. Am J 
Hypertens 4: 786, 1991.

5) Irony I, Kater CE, Biglieri EG, Shackleton CHL. Correctable subsets of primary aldosteronism. Primary adrenal hyperplasia and renin responsive adenoma. Am J Hypertens 3: 576, 1990.

6) Young WF Jr, Hogan MJ, Klee GG, Grant CS, van Heerden JA. Primary aldosteronism: diagnosis and treatment. Mayo Clin Proc 65: 96, 1990.

7) Matsuda A, Beniko M, Ikota A, et al. Primary aldosteronism with bilateral multiple aldosterone-producing adrenal adenoma. Intern Med 35: 970, 1996. 